\title{
NLO and NNLO Low Energy Constants for SU(3) Chiral Perturbation Theory
}

\author{
R.D. Mawhinney* and D.J. Murphy for the RBC-UKQCD Collaboration ${ }^{\dagger}$ \\ Department of Physics, Columbia University, New York, NY 10027, USA \\ E-mail: rdmephysics . columbia.edu, dmurphy@phys . columbia.edu
}

\begin{abstract}
We have performed global fits of pseudoscalar masses and decay constants, from a variety of RBC-UKQCD domain wall fermion ensembles, to $S U(3)$ partially quenched chiral perturbation theory at NNLO. We have determined values for 9 NLO and 10 linearly independent combinations of NNLO partially quenched low energy constants, some of which we compare to other lattice and phenomenological determinations. We discuss the convergence of the expansion and compare our current fits to earlier fits using only a subset of the current data.
\end{abstract}

The 33rd International Symposium on Lattice Field Theory

14 -18 July 2015

Kobe International Conference Center, Kobe, Japan

\footnotetext{
* Speaker.

${ }^{\dagger}$ The numerical results of QCD simulations used in these fits were produced on the QCDOC and BG/Q computers at Brookhaven National Lab and the University of Edinburgh and on the BG/Q computers at the ALCF at Argonne National Lab and Lawrence Livermore National Laboratory. These computers are supported by the U.S. Department of Energy, the RIKEN-BNL Research Center, and the U.K. DiRAC program. We thank J. Bijnens for Fortran programs implementing the NNLO PQ $\chi$ PT formulae.
} 


\section{Introduction}

During the last decade, the RBC and UKQCD Collaborations have generated a series of $2+1$ flavor domain wall fermion (DWF) and Mobius domain wall fermion (MDWF) ensembles with ever-decreasing light quark masses and larger physical volumes. Recently, $(5.5 \mathrm{fm})^{3}$ ensembles with essentially physical light and strange quark masses have been produced [1]. In 2008 [2], both $\mathrm{SU}(2)$ and $\mathrm{SU}(3)$ chiral perturbation theory $(\chi \mathrm{PT})$ fits to pseudoscalar data were used to extrapolate to the chiral limit. The pseudoscalar masses in this case were in the range of 250 to $420 \mathrm{MeV}$ and it was found that the $\mathrm{SU}(3)$ fits were not reliable. A very low value for $f_{0}$ (the $\mathrm{SU}(3)$ decay constant in the chiral limit) was found, leading to NLO terms at $\sim 400 \mathrm{MeV}$ that were $70 \%$ the size of the LO contributions and indicating an unreliable expansion. We also found that SU(3) $\chi$ PT did not fit our data when the quark masses were near the physical strange quark mass. This led us to use SU(2) fits for the physical results in [2] and all subsequent work. As ensembles with smaller light quark masses were used, the predictions for $f_{\pi}$ and $f_{K}$, using SU(2) $\chi \mathrm{PT}$, were in better and better agreement with experiment, as shown in Figure 1. With the availability of essentially physical mass ensembles, the SU(2) $\chi \mathrm{PT}$ extrapolations only involve changes in the quark masses of a few percent.

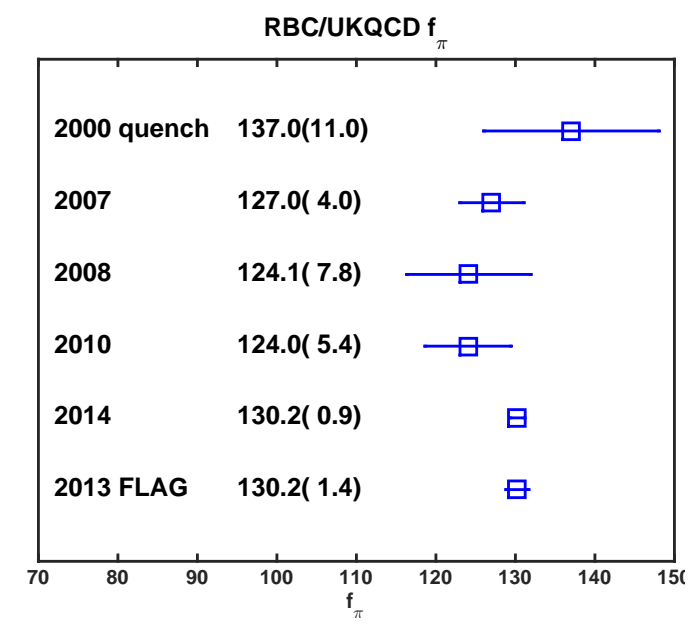

(a) $f_{\pi}$

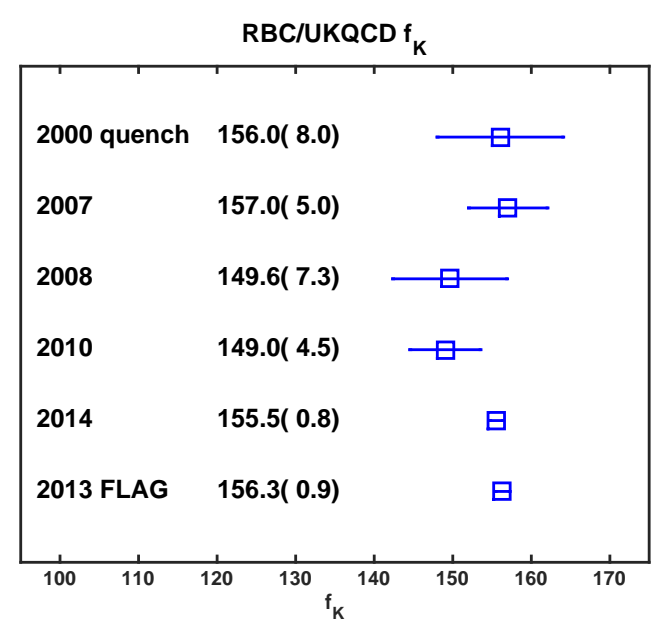

(b) $f_{K}$

Figure 1: The evolution of predictions for $f_{\pi}$ and $f_{k}$ by the RBC and UKQCD Collaborations.

In this project, we have revisited partially quenched SU(3) $\chi$ PT fits to our pseudoscalar masses and decay constants, fitting both to NLO and NNLO $\chi \mathrm{PT}$ and using the much larger range of quark masses we now have. A companion project $[3,4]$ reports our results for SU(2) NLO and NNLO fits. While $\chi$ PT fits are less important now in getting accurate results for pseudoscalar masses and decay constants at physical quark masses, it remains an important question to know how well the SU(3) $\chi$ PT series, to a given order, represents our data and determining the resulting LEC's is an important output from lattice simulations. Our DWF ensembles, with their preservation of the continuum chiral symmetries at finite lattice spacing, provide an ideal venue for testing $\chi$ PT fits. In the remainder of this report, we describe our ensembles, our fitting approach and some of our results. 


\section{Ensembles and Fit Strategy}

A list of the ensembles used in these fits is given in [3]. Here we note that these $2+1$ flavor ensembles span volumes from $(2.0 \mathrm{fm})^{3}$ to $(5.5 \mathrm{fm})^{3}, m_{\pi} L$ values from 3.8 to 5.8 , and unitary pion masses from 120 to $430 \mathrm{MeV}$. In total, we have $\sim 100$ light quark mass combinations (many partially quenched) for pseudoscalars with masses below $510 \mathrm{MeV}$. Our measured masses and decay constants have statistical errors in the 0.1 to $0.4 \%$ range.

We fit pseudoscalar masses and decay constants to NLO and NNLO SU(3) $\chi$ PT formula, with the NNLO formula encapsulated in Fortran programs from Bijnens [5]. We choose the physical values for $m_{\pi}, f_{\pi}$ and $m_{\Omega}$ to set the lattice scale and determine the (degenerate) light quark masses and the strange quark mass. For $m_{\Omega}$, we use a simple linear fit function for the light (dynamical) quark mass dependence (there are no chiral logarithms in $m_{\Omega}$ ) and separate linear fit functions for the dynamical and valence strange quark mass dependence. Note that this choice of physical quantities to set the scale differs from our choice for SU(2), where $m_{\pi}, m_{K}$ and $m_{\Omega}$ are used. Since we will be testing SU(3) $\chi \mathrm{PT}$ and probing the series as the quark mass cutoff is raised, we wanted to have our scale setting independent of quarks with masses approaching the strange quark mass scale.

As discussed extensively in [6], the 3 lattice quantities we use to match to physical values have no $O\left(a^{2}\right)$ errors. For our SU(2) fits, this means $m_{\pi}, m_{K}$ and $m_{\Omega}$ have no $O\left(a^{2}\right)$ errors, and we include an $O\left(a^{2}\right)$ term in our fits for $f_{\pi}$ and $f_{K}$. The results of the fits reported in [3], show that scaling errors for SU(2) fits are no larger than $1 \%$ for the DWF+ID ensembles and $2 \%$ for the DWF+I ensembles. For the SU(3) fits discussed here, we have not included any $O\left(a^{2}\right)$ correction term, for a few reasons. First, since we are using $m_{\pi}$ and $f_{\pi}$ as part of the scale setting, and these are related to $m_{K}$ and $f_{K}$ via $\mathrm{SU}(3) \chi \mathrm{PT}$, having no $O\left(a^{2}\right)$ terms at physical light quark masses and scaling violations at strange quark mass scales implies a cancellation of $O\left(a^{2}\right)$ terms between different orders of the chiral expansion. Secondly, our SU(2) results show that these effects are small. Finally, these are first fits to NLO and NNLO for our SU(3) data, and they are complicated enough without this extra term. In the future, such effects could, in principle, be included.

We perform our fits to SU(3) using a variant of the RBC and UKQCD Collaborations's global fitting strategy [6]. In this approach, once the fit forms are specified, an uncorrelated fit is done via least squares $\chi^{2}$ minimization. (Correlated fits are not possible, due to the large number of data being fit and the strong correlations between the partially quenched data measured on a given ensemble.) In addition to the $\chi$ PT LEC's, there are fit parameters for each ensemble's lattice spacing, relative to a reference ensemble, and light and strange quark renormalization factors. We use two pseudoscalar mass cutoffs for our fits, $370 \mathrm{MeV}$ and 510, and these will help us to understand the behavior of the series as the mass cutoff is varied.

\section{SU(3) Fits}

In this report, we describe two fits to our data, one using NLO partially quenched $\mathrm{SU}(3) \chi \mathrm{PT}$ including all pseudoscalars with mass below $370 \mathrm{MeV}$ and another using partially quenched NNLO $\mathrm{SU}(3) \chi \mathrm{PT}$ with pseudoscalar masses up to $510 \mathrm{MeV}$. We note that when we increase the cutoff from 370 to $510 \mathrm{MeV}$ and add NNLO terms to the fits, the LO constants ( $f_{0}$ and $\left.B_{0}\right)$ change by $-5 \%$ 
and $11 \%$, respectively. This indicates either instability in the expansion or insufficient data to fully stabilize the fit. To proceed, we have used the LO values for $f_{0}$ and $B_{0}$ from the NLO fit, jackknife block by jacknife block, in the NNLO fit.

Figure 2 shows the deviation between our fit function and the data. With our uncorrelated fits, we cannot quote a statistically relevant goodness of fit metric and, for an approximate representation of the data, such as $\chi$ PT to some order, the percentage deviation, which does not increase with smaller statistical errors, is useful for assessing the quality of the fits. We see that the NLO fits are accurate at about $1 \%$ and the NNLO are reliable to about $2 \%$.

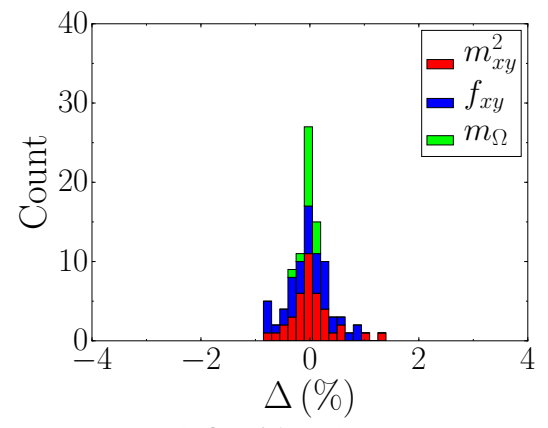

(a) NLO fit with $370 \mathrm{MeV}$ cut

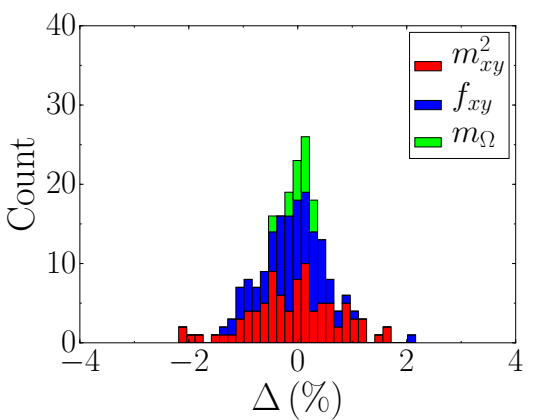

(b) NNLO fit with $510 \mathrm{MeV}$ cut

Figure 2: Stacked histograms showing the percent deviation between the data and our fits.

Figures 3 and 4 show results for $m_{\mathrm{PS}}^{2}$ and $f_{\mathrm{PS}}$ versus the quark mass. (Data with open symbols (visible by zooming) are not included in the fits.) We note the linearity in $m_{\mathrm{PS}}^{2}$ and that this implies a near cancellation between NLO and NNLO terms in the NNLO fits to masses. In particular, at the physical point the expansion is $m_{K}^{2} /\left(\chi_{l}+\chi_{h}\right) / 2=1.000-0.130(43)+0.090(41)$, where the three terms are the LO, NLO and NNLO contributions. We also have $f_{K} / f_{0}=1.000+0.315(3)+$ $0.035(30)$, showing that the NLO + NNLO terms are a 35\% correction to the LO term. These fits show that the terms in the expansion are decreasing, indicating reasonable reliability at this order.

\section{Fit Results}

Figure 5 shows our results for the LO LEC's and Figure 6 shows results for the 4 NLO LEC's that enter in $m_{\mathrm{PS}}^{2}$ and $f_{\mathrm{PS}}$ at NLO order. We see good consistency between the NLO LEC's from the NLO and NNLO fit. Also shown are previous lattice results and some continuum estimates. (B/E is Bijnens/Ecker 2014, B/J is Bijnens/Jemos 2012, G/L is Gasser/Leutwyler 1984.) As mentioned, we have results for 9 NLO and 10 linearly independent combinations of NNLO LEC's. These will be detailed in [7]. The gray bands are an estimate of our total errors.

An important part of this work is to assess whether SU(3) $\chi \mathrm{PT}$ is doing more than fitting the data - is it determining the correct LEC's and not just parameters that allow complicated formula to well approximate the data? We can gain some information by converting the SU(3) LEC's from our fits to SU(2) LEC's, since the SU(2) $\chi$ PT series is more reliable, due to smaller higher order corrections. Figures 7 and 8, show SU(2) LEC's coming from our SU(3) LEC's and the one-loop relation between $\mathrm{SU}(2)$ and $\mathrm{SU}(3)$. These are the NLO, NNLO and prediction (which includes an estimate of our errors) values shown. We also show $\mathrm{SU}(2)$ fit results from [4]. We find good 


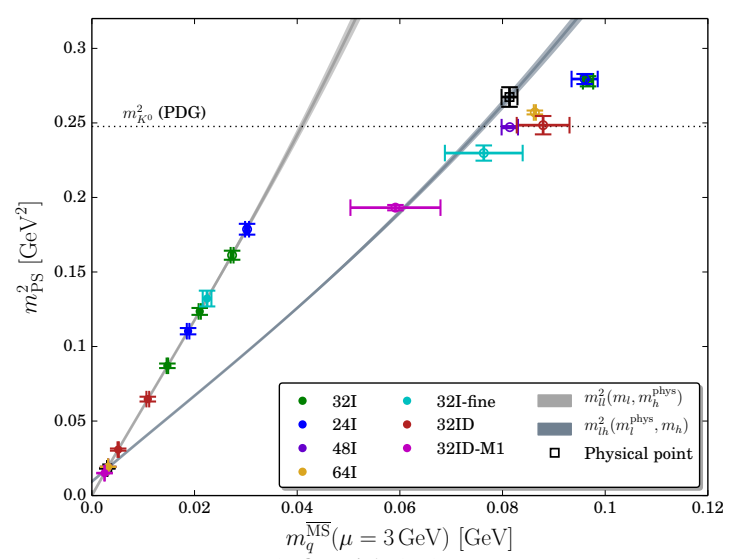

(a) NLO fit with $370 \mathrm{MeV}$ cut

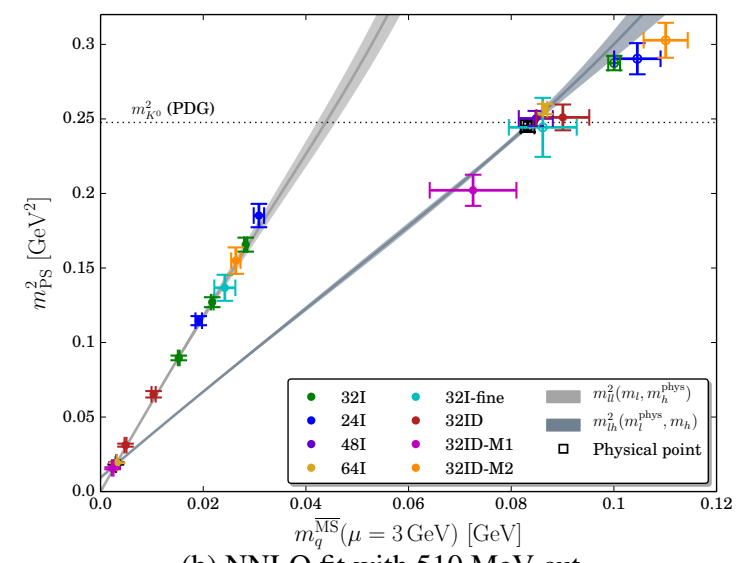

(b) NNLO fit with $510 \mathrm{MeV}$ cut

Figure 3: Unitary $m_{\mathrm{PS}}^{2}$ versus $m_{q}$. The left curve in each panel is for a degenerate pion versus the light quark mass and the right is for a kaon with a physical light quark mass, plotted versus the strange quark mass.

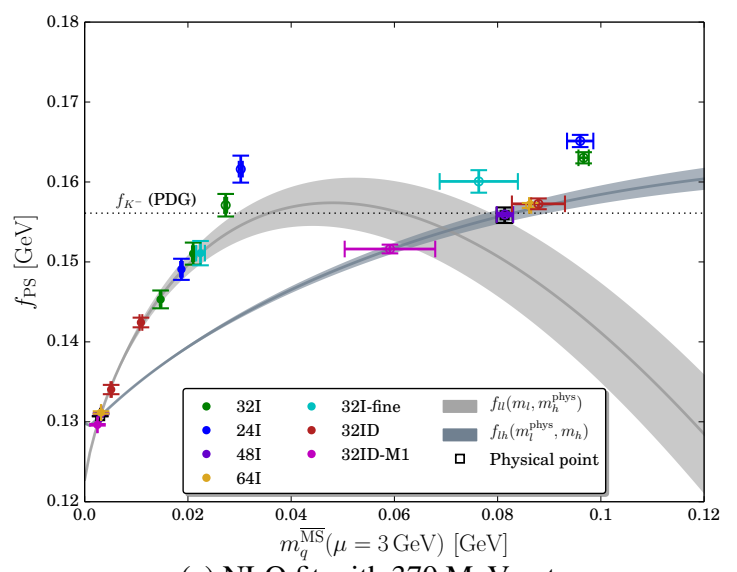

(a) NLO fit with $370 \mathrm{MeV}$ cut

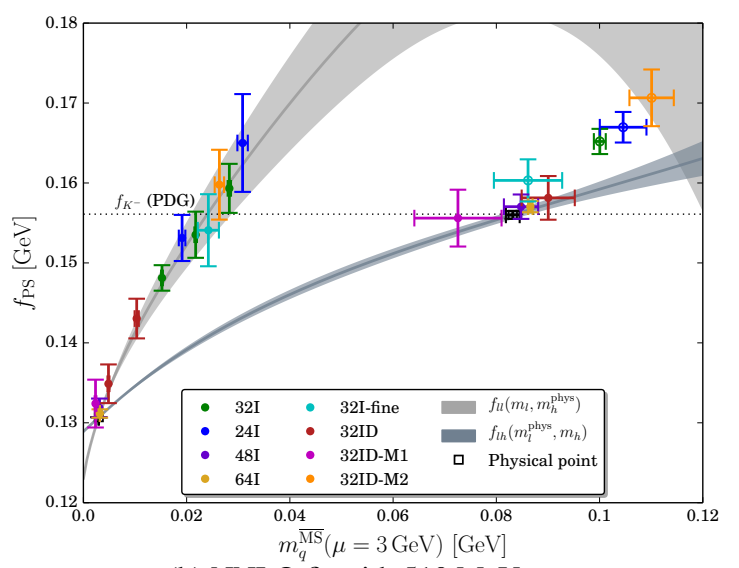

(b) NNLO fit with $510 \mathrm{MeV}$ cut

Figure 4: The same as Figure 3, except the unitary pseudoscalar decay constants are shown.

agreement with the LEC's from the direct fit to SU(2). (We note that in NNLO fits without frozen LO LEC's (not shown here, but presented in [7]), noticeable changes in LO and NLO SU(3) LEC's compensate to produce minimal changes in the SU(2) LEC's.)

As mentioned in the introduction, NLO SU(3) $\chi$ PT fits were not reliable when limited to the subset of this data available in 2008 on $24^{3}$ ensembles. To investigate this further, in light of the current, more reliable fits presented here, we have systematically removed data with lighter quarks from our $\mathrm{SU}(3)$ fits. Figure 9 a shows the fits to $f_{\mathrm{PS}}$ when all ensembles are used (black lines) and only the $24^{3}$ ensemble of [2] is used (red lines). The solid lines show the extrapolation in the light quark mass with the strange quark mass fixed at its physical value and the dashed lines are the SU(3) symmetric case. One sees how only fitting the blue data points (and their unshown partially quenched companions) gives an extrapolation that deviates markedly from the fits with more data. In Figure 9b, we have extracted the contributions of the $24^{3}$ data to the fit to all ensembles. One sees that one of the ensembles is systematically above the fit and the other systematically below. 


\begin{tabular}{|c|}
\hline NLO fit \\
NNLO fit \\
\hline Prediction \\
\hline MILC (2010) \\
MILC (2009A) \\
MILC (2009) \\
\hline
\end{tabular}
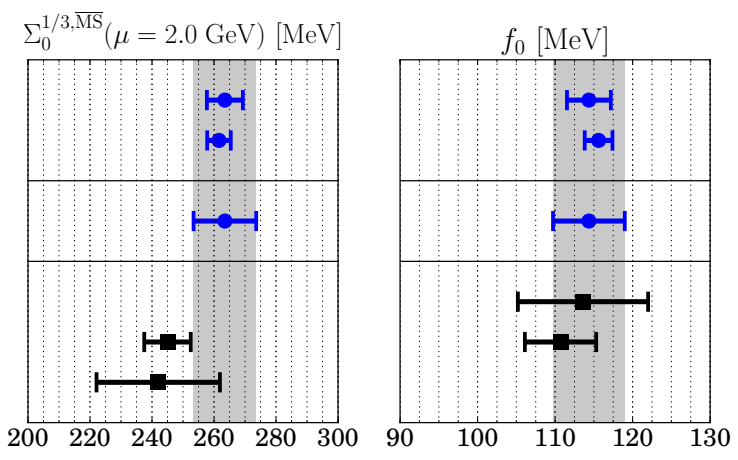

Figure 5: Results for SU(3) LO LEC's.
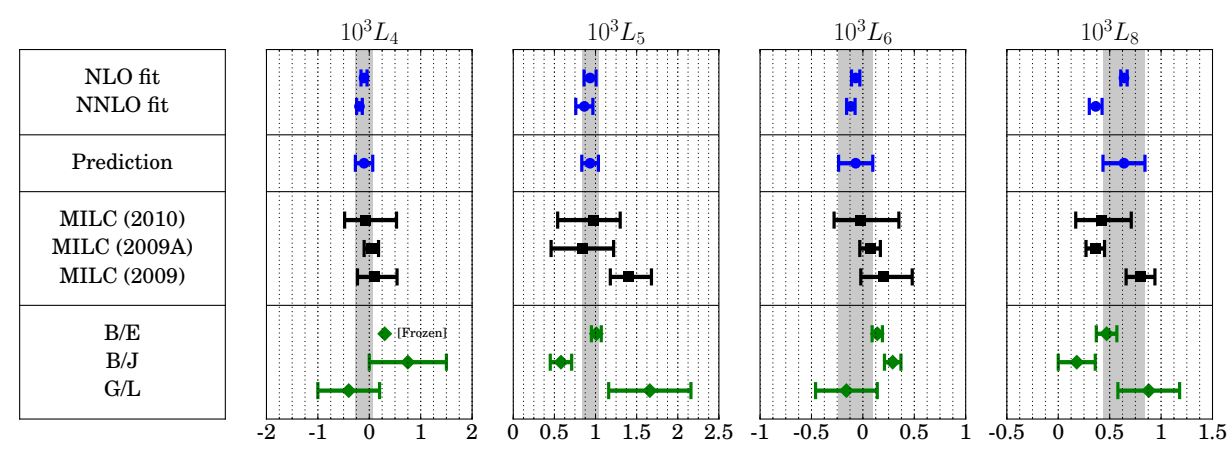

Figure 6: Results for some SU(3) NLO LEC's.
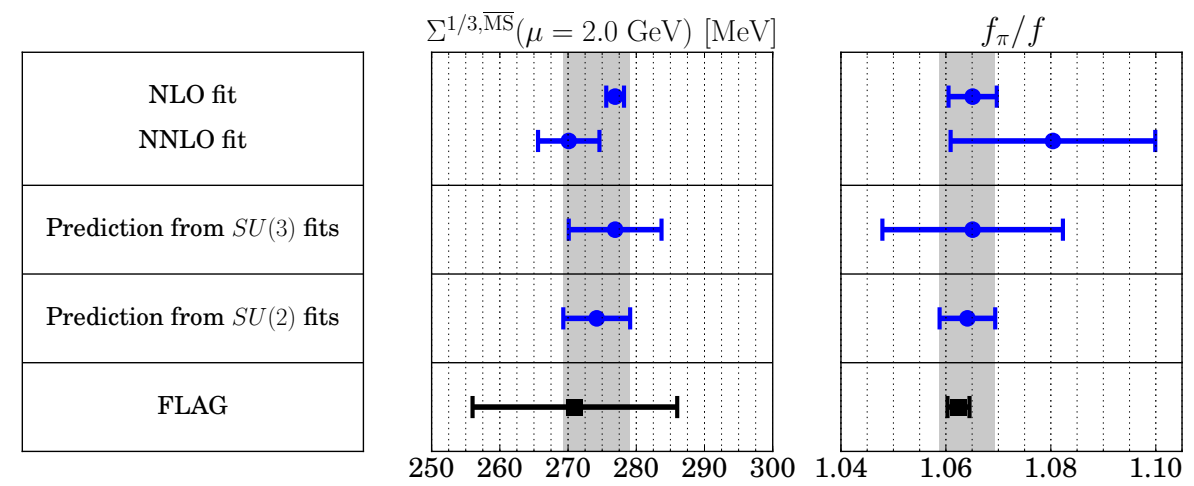

Figure 7: Results for LO SU(2) LEC's from our SU(3) fits and the one-loop relations between SU(2) and SU(3) LEC's.

\section{Conclusions}

We have performed fits of our data to partially quenched SU(3) $\chi \mathrm{PT}$ at NLO order with a $370 \mathrm{MeV}$ pseudoscalar mass cut, and at NNLO order with a $510 \mathrm{MeV}$ pseudoscalar mass cut. We find the fits represent our data at the $1 \%$ level for NLO and the $2 \%$ level for NNLO. The LEC's we extract are consistent with other determinations and the SU(2) LEC's we find, from one-loop conversion from our SU(3) results, are in agreement with our direct fits to SU(2) $\chi$ PT. We find the NLO and NNLO series to have reasonably sized corrections, relative to the LO results. 

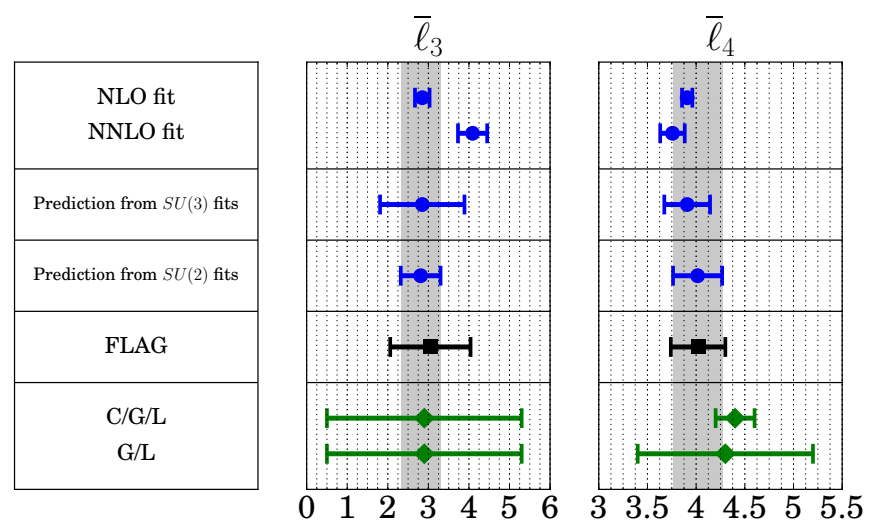

Figure 8: The same as Figure 7, except for two SU(2) NLO LEC's.

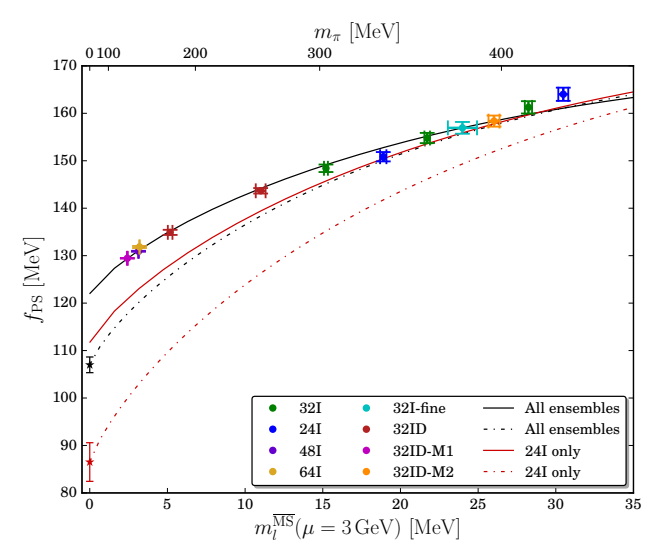

(a) SU(3) NLO fit with all data and $24^{3}$ data

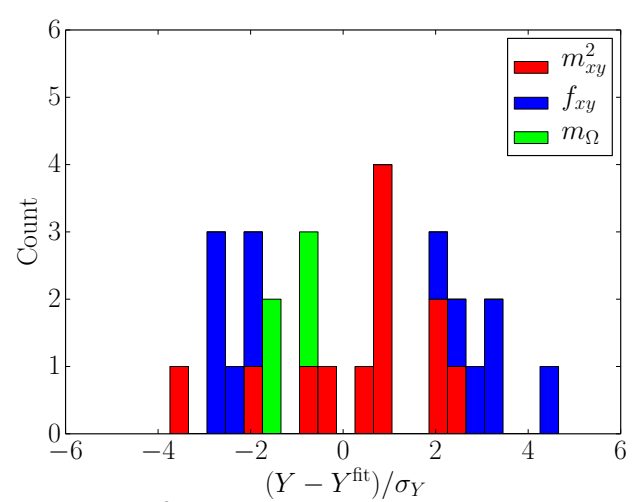

(b) $24^{3}$ data contribution to all data fit

Figure 9: Results from comparing a NLO fit to all the data to a NLO fit only using data from [2].

\section{References}

[1] T. Blum, et. al., RBC and UKQCD Collaborations, Domain wall QCD with physical quark masses, arXiv:1411.7017.

[2] C. Allton, et. al., RBC and UKQCD Collaborations, Physical Results from 2+1 Flavor Domain Wall QCD and SU(2) Chiral Perturbation Theory, Phys. Rev. D78 (2008) 114509, [arXiv:0804.0473].

[3] D. Murphy and R. Mawhinney, NLO and NNLO Low Energy Constants for SU(2) Chiral Perturbation Theory, in the proceedings of Lattice 2015, POS (Lattice 2015) 061.

[4] P.A. Boyle, et. al., RBC and UKQCD Collaborations, The Low Energy Constants of SU(2) Partially Quenched Chiral Perturbation Theory from $N_{f}=2+1$ Domain Wall QCD, arXiv:1511.01950.

[5] J. Bijnens, N. Danielsson, and T. Lahde, Three-flavor partially quenched chiral perturbation theory at NNLO for meson masses and decay constants, Phys. Rev. D73 (2006) 074509, [hep-lat/0602003].

[6] Y. Aoki, et. al., RBC and UKQCD Collaborations, Continuum Limit Physics from 2+1 Flavor Domain Wall QCD, Phys. Rev. D83 (2011) 074508, [arXiv:1011.0892].

[7] C. Kelly, R. Mawhinney and D. Murphy, The Low Energy Constants of SU(3) Partially Quenched Chiral Perturbation Theory from $N_{f}=2+1$ Domain Wall $Q C D$, in preparation. 